\title{
A software package for Mori dream spaces
}

\author{
Jürgen Hausen and Simon Keicher
}

\begin{abstract}
Mori dream spaces form a large example class of algebraic varieties, comprising the well-known toric varieties. We provide a first software package for the explicit treatment of Mori dream spaces and demonstrate its use by presenting basic sample computations. The software package is accompanied by a Cox ring database which delivers defining data for Cox rings and Mori dream spaces in a suitable format. As an application of the package, we determine the common Cox ring for the symplectic resolutions of a certain quotient singularity investigated by Bellamy-Schedler and Donten-Bury-Wiśniewski.
\end{abstract}

\section{Introduction}

By a Mori dream space we mean here a normal complete variety $X$ defined over an algebraically closed field $\mathbb{K}$ of characteristic zero having a finitely generated divisor class group $\mathrm{Cl}(X)$ and a finitely generated Cox ring

$$
\mathcal{R}(X)=\bigoplus_{\mathrm{Cl}(X)} \Gamma(X, \mathcal{O}(D))
$$

where we refer to [2] for the details of the definition. Mori dream spaces have been introduced by $\mathrm{Hu}$ and Keel [19] as a class of varieties with an optimal behaviour with respect to the minimal model program. Well-known examples of Mori dream spaces are toric and, more generally, spherical varieties, smooth Fano varieties [8] or Calabi-Yau varieties with a polyhedral effective cone [27]. Examples of general type can be obtained by Lefschetz-type theorems [1, 21].

An important feature of Mori dream spaces is that they allow an explicit encoding in terms of algebraic and combinatorial data $[\mathbf{2}, \mathbf{6}, \mathbf{1 5}]$ and their theory has close relations to toric geometry. This turns Mori dream spaces into natural candidates for extending the 'testing ground of algebraic geometry' given by the toric varieties. For working explicitly with toric varieties, there are meanwhile several software packages available, such as $[\mathbf{7}, \mathbf{9}, \mathbf{1 4}, \mathbf{2 2}, \mathbf{2 3}, \mathbf{2 8}]$. Our intention is to provide with MDSpackage [17] such a tool also for the larger class of Mori dream spaces.

Let us give a more concrete impression. Every Mori dream space $X$ is encoded by its Cox ring $\mathcal{R}(X)$ plus data located in the divisor class group $\mathrm{Cl}(X)$ which fix the isomorphy type among all varieties sharing $\mathcal{R}(X)$ as Cox ring. For instance, we can define a three-dimensional projective Mori dream space $X$ by prescribing its divisor class group as $K:=\mathbb{Z}^{2}$, its $K$-graded Cox ring as

$$
\begin{gathered}
R:=\mathbb{K}\left[T_{1}, \ldots, T_{6}\right] /\left\langle T_{1} T_{2}+T_{3} T_{4}+T_{5}^{2}+T_{6}^{2}\right\rangle, \\
Q:=\left[\begin{array}{rrrrrr}
-2 & 2 & -1 & 1 & 0 & 0 \\
1 & 1 & 1 & 1 & 1 & 1
\end{array}\right]
\end{gathered}
$$

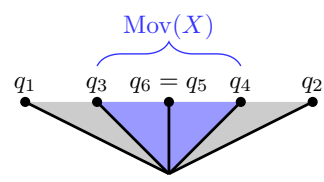

Received 14 August 2014; revised 22 July 2015.

2010 Mathematics Subject Classification 14Q10, 14Q15 (primary).

The second author was partially supported by the DFG Priority Program SPP 1489. 
where $Q$ has the generator degrees $\operatorname{deg}\left(T_{i}\right) \in K$ as its columns, and an ample class $w \in K$ taken from the relative interior of the above blue cone, that is, the prospective moving cone of $X$. The $K$-grading of $R$ defined by $Q$ gives rise to an action of the 2 -torus $H=\left(\mathbb{K}^{*}\right)^{2}$ on

$$
\bar{X}=V\left(T_{1} T_{2}+T_{3} T_{4}+T_{5}^{2}+T_{6}^{2}\right) \subseteq \mathbb{K}^{6}
$$

and the Mori dream space $X$ is the quotient by $H$ of the set $\widehat{X} \subseteq \bar{X}$ of semistable points associated to the weight $w$. As mentioned, the divisor class group and Cox ring of $X$ are given by $\mathrm{Cl}(X)=K$ and $\mathcal{R}(X)=R$. The task of MDSpackage is then to extract further geometric invariants and properties from these defining data. For example, it determines the Picard group and the singularities of $X$ as

$$
\begin{gathered}
\operatorname{Pic}(X)=6 \mathbb{Z} \oplus 3 \mathbb{Z} \subseteq \mathbb{Z}^{2}=\mathrm{Cl}(X), \\
\operatorname{Sing}(X)=\{\{1,5,6\},\{1,2,5,6\},\{1,2,6\},\{2,3\},\{1,4\},\{1,2,5\}\},
\end{gathered}
$$

where the output on the singularities provides information on their Cox coordinates; for example, $\{1,5,6\}$ says that there is a singular point $x \in X$ stemming from a point $z \in \widehat{X} \subseteq \mathbb{K}^{6}$ having precisely $z_{1}, z_{5}, z_{6}$ as non-zero coordinates.

MDSpackage aims to be an easy-to-use computing environment for up to medium-size computations. In the subsequent section, we present sample computations and thereby explain the syntax. The major computational ingredients come from commutative algebra and polyhedral combinatorics. The basic features of MDSpackage are:

- basics on finitely generated abelian groups and algebras graded by them;

- computing Picard group, local class groups, cones of effective, movable or semiample divisor classes, Mori chamber decomposition, pullback of $\mathbb{Q}$-Cartier divisors;

- computing the canonical toric ambient variety, induced orbit stratification, irrelevant ideal;

- testing (quasi-)smoothness, $(\mathbb{Q}$-)factoriality, completeness, (quasi-)projectivity;

- computing the singular locus, global resolution of singularities (approved for varieties with torus action of complexity one, experimental in the general case);

- for complete intersection Cox rings: computing intersection numbers, graph of exceptional divisors, anticanonical divisor class, Gorenstein index, testing $(\mathbb{Q}$-)Gorenstein and Fano properties;

- for varieties with a torus action of complexity one: tests for being $(\varepsilon-\log )$ terminal, almost homogeneous, computing roots of the automorphism group.

Detailed background on the algorithms and a complete manual for MDSpackage can be found in [25]. Moreover, a comprehensive online manual is available at [17]. Our package is implemented in the computer algebra system Maple [26] and makes use of the convex package by Franz $[\mathbf{1 3}]$.

MDSpackage has been essentially used in the classification of Fano varieties. In [4] the $\mathbb{Q}$-factorial terminal Fano threefolds of Picard number one with an effective action of a twodimensional torus are classified; among other computations performed with MDSpackage, this involves more than $10^{6}$ terminality tests for possible candidates. Other applications are the classification results on $\mathbb{K}^{*}$-surfaces of high Gorenstein index given in [25].

In order to open a broad computer-supported access to Mori dream spaces, we are building up a database of Cox rings [16], the entries of which can be exported in a suitable data format for MDSpackage. Our motivation for creating such a combined toolkit is the fruitful linking of the theory of weighted complete intersections with the graded ring database; see [10, 20].

In Section 3 we apply MDSpackage to continue work of Bellamy-Schedler [5] and DontenBury-Wiśniewski [12] on the 81 symplectic resolutions of a certain quotient singularity: in Theorem 3.2 we determine the common Cox ring of these resolutions. 


\section{Working with MDSpackage}

According to $[\mathbf{6}, \mathbf{1 5}]$, a Mori dream space $X$ is encoded by a bunched ring. This basically is an integral algebra $R=\bigoplus_{K} R_{w}$ graded by a finitely generated abelian group $K$ such that the $K$-homogeneous elements admit unique factorization together with a collection $\Phi$, called 'bunch', of convex polyhedral cones in $K \otimes \mathbb{Q}$. Concretely, the $K$-graded algebra $R$ is given by generators and relations and a degree map assigning to each generator its $K$-degree:

$$
R=\mathbb{K}\left[T_{1}, \ldots, T_{r}\right] /\left\langle g_{1}, \ldots, g_{s}\right\rangle, \quad Q: \mathbb{Z}^{r} \rightarrow K, \quad e_{i} \mapsto \operatorname{deg}\left(T_{i}\right) .
$$

The bunch of cones $\Phi$ fixes the isomorphy type of $X$ among all varieties having $R$ as Cox ring. In case of a projective Mori dream space $X$, one can simply define $\Phi$ by fixing an ample class $w \in K$ from inside the moving cone.

We now demonstrate the practical work with MDSpackage [17] by means of three example computations; more can be found in [25].

EXAMPLE 2.1. The first step in defining a Mori dream space $X$ with MDSpackage is to enter the abelian groups $E:=\mathbb{Z}^{r}, K$ and the degree map $Q: E \rightarrow K$ :

$>\mathrm{E}:=\operatorname{createAG}(8)$;

$$
E:=A G(8,[])
$$

$>\mathrm{K}:=\operatorname{createAG}(3,[2])$;

$$
\begin{gathered}
K:=A G(3,[2]) \\
>\text { A := cols2matrix }([[1,0,1,1],[1,1,1,0],[0,1,1,1],[0,-1,1,0], \\
[-1,-1,1,1],[-1,0,1,0],[2,1,1,1],[-2,-1,1,0]]) ; \\
A:=\left[\begin{array}{rrrrrrrr}
1 & 1 & 0 & 0 & -1 & -1 & 2 & -2 \\
0 & 1 & 1 & -1 & -1 & 0 & 1 & -1 \\
1 & 1 & 1 & 1 & 1 & 1 & 1 & 1 \\
1 & 0 & 1 & 0 & 1 & 0 & 1 & 0
\end{array}\right] \\
>\mathrm{Q}:=\operatorname{createAGH}(\mathrm{E}, \mathrm{K}, \mathrm{A}) ; \\
Q:=\operatorname{AGH}([8,[]],[3,[2]]) .
\end{gathered}
$$

Here we took $E=\mathbb{Z}^{8}$ and $K=\mathbb{Z}^{3} \oplus \mathbb{Z} / 2 \mathbb{Z}$ and the $4 \times 8$ matrix $A$ fixes a map $\mathbb{Z}^{8} \rightarrow \mathbb{Z}^{4}$ inducing the degree map $Q: E \rightarrow K$. The next step is to define the $K$-graded ring $R$. We have to specify variables, relations and the grading:

$$
\begin{aligned}
& >\mathrm{TT}:=\operatorname{vars}(8) ; \\
& \qquad T:=[T[1], T[2], T[3], T[4], T[5], T[6], T[7], T[8]] \\
& >\mathrm{RL}:=[\mathrm{T}[1] * \mathrm{~T}[6]+\mathrm{T}[2] * \mathrm{~T}[5]+\mathrm{T}[3] * \mathrm{~T}[4]+\mathrm{T}[7] * \mathrm{~T}[8]] ; \\
& R L:=[T[1] T[6]+T[2] T[5]+T[3] T[4]+T[7] T[8]] \\
& >\mathrm{R}:=\operatorname{createGR}(\mathrm{RL}, \mathrm{TT},[\mathrm{Q}], \quad \text { 'nocheck' }) ; \\
& R:=G R(8,1,[3,[2]]) .
\end{aligned}
$$


The last output line indicates that $R$ is given by eight generators and one relation and its grading group is isomorphic to $\mathbb{Z}^{3} \oplus \mathbb{Z} / 2 \mathbb{Z}$; the option 'nocheck' speeds up the computation by omitting plausibility checks. To define $X$, it remains to fix a prospective ample class $w \in K \otimes \mathbb{Q}$ :

$>\mathrm{w}:=[0,0,2]$;

$$
w:=[0,0,2]
$$

$>\mathrm{X}:=\operatorname{createMDS}(\mathrm{R}, \mathrm{w})$;

$$
X:=\operatorname{MDS}(8,1,4,[3,[2]]) .
$$

The last output line is similar to that for $R$, the only new thing is the third entry, saying that the resulting Mori dream space $X$ is of dimension four. We are now ready for computations with $X$. First, we determine the Picard group of $X$ as a subgroup of $\mathrm{Cl}(X)=K$ and the factor group $\mathrm{Cl}(X) / \operatorname{Pic}(X)$ :

$>$ Pic := MDSpic $(X)$;

$$
\text { Pic := } A G(3,[])
$$

$>\operatorname{AGfactgrp}(\mathrm{K}, \mathrm{Pic})$;

$$
A G(0,[2,12,12,24]) .
$$

The first output tells us that $\operatorname{Pic}(X)$ is of rank three and torsion free, that is, we have $\operatorname{Pic}(X) \cong$ $\mathbb{Z}^{3}$. The second output says that

$$
\mathrm{Cl}(X) / \operatorname{Pic}(X) \cong \mathbb{Z} / 2 \mathbb{Z} \oplus \mathbb{Z} / 12 \mathbb{Z} \oplus \mathbb{Z} / 12 \mathbb{Z} \oplus \mathbb{Z} / 24 \mathbb{Z} .
$$

The cones of semiample, movable and effective divisor classes in the rational divisor class group $K \otimes_{\mathbb{Z}} \mathbb{Q}$ are computed as follows:

$>$ MDSsample $(\mathrm{X})$;

$$
\operatorname{CONE}(3,3,0,8,8)
$$

$>\operatorname{MDSmov}(\mathrm{X})$;

$$
\text { CONE }(3,3,0,4,4)
$$

$>\operatorname{MDSeff}(\mathrm{X})$;

$$
\text { CONE }(3,3,0,4,4) \text {. }
$$

These cones are stored in convex format. In particular, for the first one, the output tells us that we have a three-dimensional cone in 3-space having zero-dimensional lineality part and eight rays and eight facets; further information on the cones can be extracted via suitable convex commands. We compute the Mori chamber decomposition of the effective cone:

$>\mathrm{F}:=\operatorname{MDSchambers}(\mathrm{X})$;

$$
F:=F A N(3,0,[0,0,37])
$$


Again the fan is stored in convex format. We refer to [19] for theoretical background of the Mori chamber decomposition and to [24] for the algorithmic aspects. We can also ask MDSpackage for a povray visualization:

$>\operatorname{render}(\mathrm{F})$;

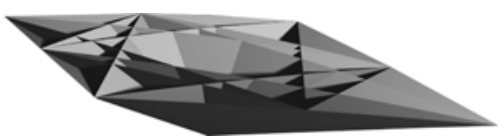

For complete intersection Cox rings like the current example, we may also check if $X$ is a Fano variety and compute its Gorenstein index:

$>\operatorname{MDSisfano}(\mathrm{X})$;

true

$>$ MDSgorensteinind $(\mathrm{X})$;

4

Example 2.2. We consider the Gorenstein del Pezzo surface $X$ having Picard number one, singularity type $E_{6} A_{2}$ and finite automorphism group. The Cox ring is given by

$$
\mathcal{R}(X)=\mathbb{K}\left[T_{1}, \ldots, T_{4}\right] /\left\langle-T_{1} T_{4}^{2}+T_{2}^{3}+T_{2} T_{3} T_{4}+T_{3}^{3}\right\rangle, \quad Q=\left[\begin{array}{cccc}
1 & 1 & 1 & 1 \\
\overline{1} & \overline{2} & \overline{0} & \overline{1}
\end{array}\right],
$$

where the divisor class group $\mathrm{Cl}(X)$ is isomorphic to $K=\mathbb{Z} \oplus \mathbb{Z} / 3 \mathbb{Z}$; see [18, Theorem 4.1]. First, we have to enter $X$ :

$>\mathrm{TT}:=\operatorname{vars}(4)$;

$$
T T:=[T[1], T[2], T[3], T[4]]
$$

$>\mathrm{RL}:=[-\mathrm{T}[1] * \mathrm{~T}[4] \wedge 2+\mathrm{T}[2] \wedge 3+\mathrm{T}[2] * \mathrm{~T}[3] * \mathrm{~T}[4]+\mathrm{T}[3] \wedge 3] ;$

$$
R L:=\left[-T[1] T[4]^{2}+T[2]^{3}+T[2] T[3] T[4]+T[3]^{3}\right]
$$

$>\mathrm{B}:=\operatorname{cols} 2$ matrix $([[1,1],[1,2],[1,0],[1,1]])$;

$$
B:=\left[\begin{array}{llll}
1 & 1 & 1 & 1 \\
1 & 2 & 0 & 1
\end{array}\right]
$$

$>Q:=\operatorname{createAGH}(\operatorname{createAG}(4), \operatorname{createAG}(1,[3]), \mathrm{B})$;

$$
Q:=A G H([4,[]],[1,[3]])
$$

$>\mathrm{R}:=\operatorname{createGR}(\mathrm{RL}, \mathrm{TT},[\mathrm{Q}])$;

$$
R:=G R(4,1,[1,[3]])
$$

$>\mathrm{X}:=\operatorname{create\operatorname {MDS}}(\mathrm{R},[1])$;

$$
X:=\operatorname{MDS}(4,1,2,[1,[3]])
$$


We take a look at the singularities of $X$ and compute a resolution. Let us check smoothness properties and determine the singular locus:

$>\operatorname{MDSissmooth}(\mathrm{X})$;

$$
\text { false }
$$

$>$ MDSisquasismooth $(\mathrm{X})$;

$$
\text { false }
$$

$>\mathrm{L}:=\operatorname{MDSsing}(\mathrm{X})$

$$
\begin{aligned}
L:= & {\left[\left[\left[-2 T[1] T[4]+T[2] T[3], T[2] T[4]+3 T[3]^{2}, 3 T[2]^{2}+T[3] T[4],-T[4]^{2},\right.\right.\right.} \\
& \left.\left.\left.-T[1] T[4]^{2}+T[2]^{3}+T[2] T[3] T[4]+T[3]^{3}\right], \quad[T[1], T[2], T[3], T[4]]\right], \quad[\{4\},\{1\}]\right] .
\end{aligned}
$$

The first entry of L is a list of generators for the ideal of the singular locus of the total coordinate space $\bar{X}$ and a list of used variables. As mentioned, the second entry tells us that the singularities are the points $[0,0,0,1]$ and $[1,0,0,0]$, given in Cox coordinates. The minimal resolution $X_{2} \rightarrow X$ is computed by

$>\mathrm{X} 2:=$ MDSresolvesing (X, 'verify', 'minimal');

Verification successful.

$$
X 2:=\operatorname{MDS}(12,1,2,[9,[]]) .
$$

Since the verification was successful, this means that $X_{2}$ is indeed a smooth Mori dream space. In accordance with $\left[\mathbf{1 1}, \mathrm{p} .40\right.$, type $\left.E_{6} A_{2}\right]$, we obtain for the Cox ring of $X_{2}$ a single defining equation and the following degree matrix:

$>\mathrm{R} 2:=\operatorname{MDSdata}(\mathrm{X} 2)[1]$;

$$
R 2:=G R(12,1,[9,[]])
$$

$>$ GRdata(R2) [1];

$$
\begin{gathered}
{\left[-T[1] T[4]^{2} T[5]+T[2]^{3} T[12] T[7]^{2} T[8]+T[2] T[3] T[11] T[4] T[5] T[6] T[7] T[8] T[10]\right.} \\
\left.+T[3]^{3} T[9] T[11]^{2} T[10]\right]
\end{gathered}
$$

$>$ GRdata (R2) [3] ;

$$
\left.\left[\begin{array}{rrrrrrrrrrrr}
1 & 1 & 1 & 1 & 0 & 0 & 0 & 0 & 0 & 0 & 0 & 0 \\
1 & 0 & 0 & -1 & 1 & 0 & 0 & 0 & 0 & 0 & 0 & 0 \\
1 & 0 & 0 & 0 & -1 & 1 & 0 & 0 & 0 & 0 & 0 & 0 \\
1 & 0 & 0 & 0 & 0 & 0 & 0 & 1 & 1 & 0 & 0 & 0 \\
0 & -1 & 0 & 0 & -1 & 0 & 1 & 0 & -1 & 0 & 0 & 0 \\
0 & 0 & 0 & 0 & 0 & -1 & 0 & 0 & -1 & 1 & 0 & 0 \\
1 & 0 & 0 & 0 & 0 & 0 & 1 & -1 & 0 & 1 & 0 & 0 \\
0 & 1 & 0 & 1 & 0 & 0 & 0 & -1 & 0 & 0 & 1 & 0 \\
0 & 0 & 0 & 0 & 1 & 0 & 0 & 0 & 1 & 0 & 0 & 1
\end{array}\right]\right]
$$


We compute the graph of exceptional curves and a list of the self-intersection numbers of the invariant divisors $V\left(X ; T_{i}\right)$; looking at the subgraph of $(-2)$-curves, we then see the $E_{6^{-}}$and the $A_{2}$-singularities:

> MDSintersgraph(X2, 'latex');
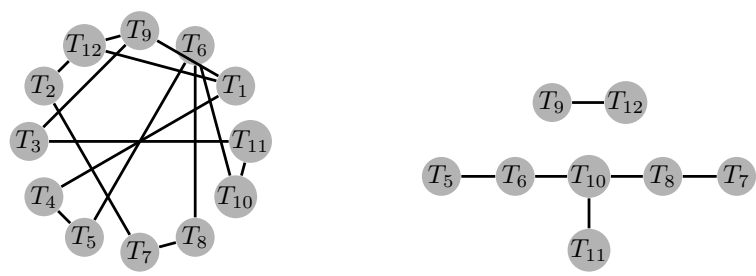

$>$ MDSintersno(X2);

$$
[-1,-1,-1,-1,-2,-2,-2,-2,-2,-2,-2,-2] .
$$

The plot of the intersection graph is provided by MDSpackage either as Maple objects or in LTEXformat.

EXAMPle 2.3. We consider a variety with torus action of complexity one. The Cox ring $R$ of any such variety $Y$ can be simply encoded by a pair $(A, P)$ of matrices; see for example [2] for details. Here is how to enter it in MDSpackage:

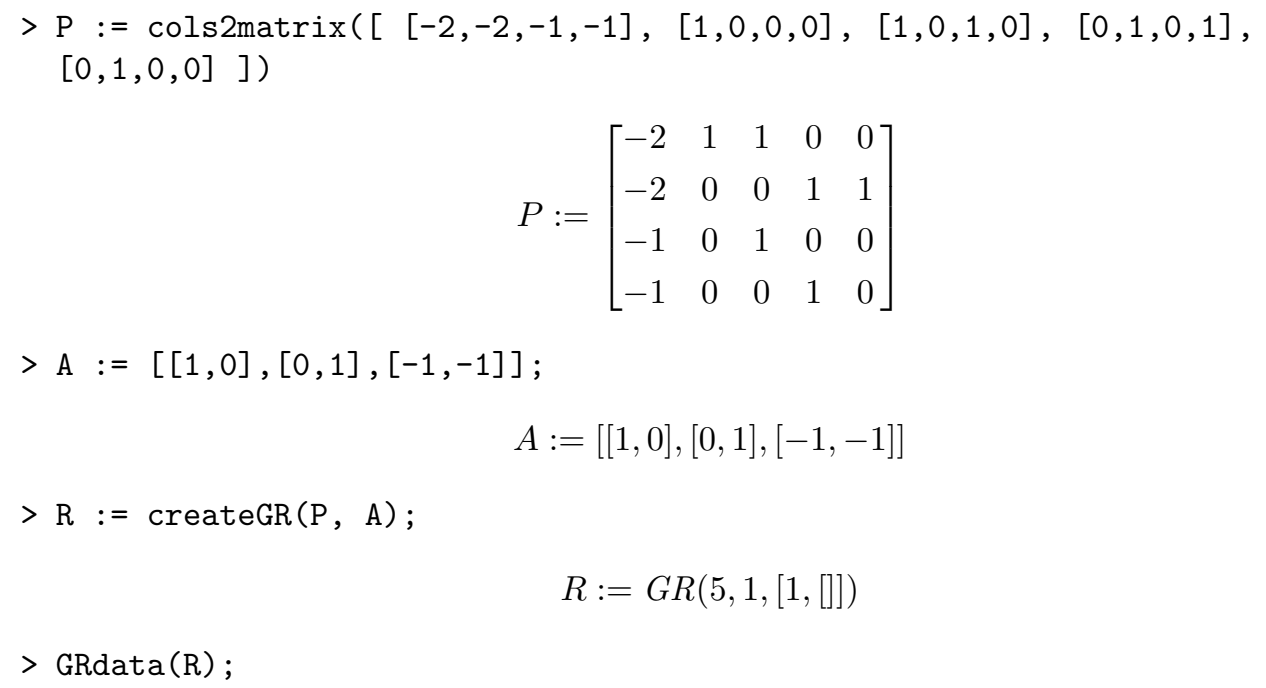

$$
\begin{gathered}
{\left[\left[T[4] T[5]+T[2] T[3]+T[1]^{2}\right],[T[1], T[2], T[3], T[4], T[5]],\right.} \\
{\left[A G H([5,[]],[1,[]]),\left[\begin{array}{lllll}
1 & 1 & 1 & 1 & 1
\end{array}\right], \ldots\right] .}
\end{gathered}
$$

So far we have defined the prospective Cox ring $R$. To specify a variety $Y$ with Cox ring $R$, we have to fix a bunch $\Phi$. However, the unit component Aut $(Y)^{0}$ of the (linear algebraic) automorphism group depends only on the Cox ring and we can compute its roots as follows:

$>\operatorname{MDSautroots}(\mathrm{R})$;

$$
\{[1,-1],[1,1],[-1,-1],[-1,1],[0,-1],[0,1],[1,0],[-1,0]\} .
$$


We have obtained the root system $B_{2}$ of the corresponding orthogonal group, as expected for the smooth quadric $Y$ in $\mathbb{P}_{5}$.

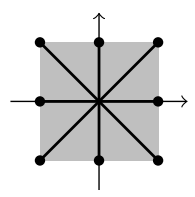

REMARK 2.4. As a support of MDSpackage, we build up the Cox ring database [16]. This database stores known Cox rings of varieties. Among others, it provides at the moment the Cox rings of all non-toric:

- smooth rational surfaces up to Picard number five;

- smooth rational surfaces of Picard number six admitting only trivial $\mathbb{K}^{*}$-actions;

- Gorenstein del Pezzo surfaces of Picard number one;

- cubic surfaces with at most rational double points;

- Gorenstein del Pezzo $\mathbb{K}^{*}$-surfaces;

- smooth Fano threefolds of Picard number at most two;

- terminal Fano threefolds of Picard number one with a two-torus action.

We use the representation in terms of bunched rings explained in Section 2: for a given Mori dream space $X$, the database stores the tuple $\left(r,\left\{g_{1}, \ldots, g_{s}\right\}, K, Q, \Phi\right)$, where the divisor class group and the Cox ring of $X$ are given by

$$
\mathrm{Cl}(X)=K, \quad \mathcal{R}(X)=\mathbb{K}\left[T_{1}, \ldots, T_{r}\right] /\left\langle g_{1}, \ldots, g_{s}\right\rangle,
$$

the entry $Q$ is the degree matrix and $\Phi$ is the bunch of cones fixing $X$; in case of a projective $X$ we just replace $\Phi$ with an ample class $w \in K$. Here are some basic features of the database:

- if available, further information is stored, such as basic geometric properties (dimension, Picard number, type of singularities, etc.), reference to author and original literature;

- the web interface [16] allows searching the Cox ring database according to keywords, authors and geometric properties;

- the stored Cox rings and varieties can be directly exported in the necessary syntax for MDSpackage or $\mathrm{LAT}_{\mathrm{E}} \mathrm{X}$.

For instance, the 40 Fano threefolds of [4] are found by entering Fano AND terminal AND Picard number one AND dimension three AND Q-factorial in the search field description. Searching the id field for 96, 97, 6 and 98 yields the Cox rings discussed in the introduction and the previous three examples.

\section{Application: resolutions of a quotient singularity}

We use MDSpackage to study a symplectic quotient singularity discussed by Bellamy and Schedler [5] and by Donten-Bury and Wiśniewski [12]. More precisely, we consider the subgroup $G \subseteq \mathrm{Gl}_{4}(\mathbb{C})$ generated by the matrices

$$
\begin{aligned}
& {\left[\begin{array}{rrrr}
1 & 0 & 0 & 0 \\
0 & -1 & 0 & 0 \\
0 & 0 & 1 & 0 \\
0 & 0 & 0 & -1
\end{array}\right], \quad\left[\begin{array}{rrrr}
0 & \mathrm{I} & 0 & 0 \\
-\mathrm{I} & 0 & 0 & 0 \\
0 & 0 & 0 & -\mathrm{I} \\
0 & 0 & \mathrm{I} & 0
\end{array}\right], \quad\left[\begin{array}{llll}
0 & 1 & 0 & 0 \\
1 & 0 & 0 & 0 \\
0 & 0 & 0 & 1 \\
0 & 0 & 1 & 0
\end{array}\right] \text {, }} \\
& {\left[\begin{array}{rrrr}
0 & 0 & 0 & 1 \\
0 & 0 & -1 & 0 \\
0 & -1 & 0 & 0 \\
1 & 0 & 0 & 0
\end{array}\right], \quad\left[\begin{array}{rrrr}
0 & 0 & 0 & \mathrm{I} \\
0 & 0 & -\mathrm{I} & 0 \\
0 & \mathrm{I} & 0 & 0 \\
-\mathrm{I} & 0 & 0 & 0
\end{array}\right]}
\end{aligned}
$$


The aim is to study resolutions of singularities of the quotient space $\mathbb{C}^{4} / G$. In Theorem 3.2, we will determine for one of them the Cox ring and show that all of its flops are as well resolutions of singularities. This continues $[\mathbf{5 , 1 2}]$ and also retrieves the 81 symplectic resolutions of $\mathbb{C}^{4} / G$ discussed there.

Observe that the commutator subgroup $[G, G] \subseteq G$ is generated by the negative unit matrix $-E_{4}$ and hence is of order two. Moreover, the abelianization $G^{\prime}:=G /[G, G]$ is isomorphic to $(\mathbb{Z} / 2 \mathbb{Z})^{4}$ and generated by the classes of the last four matrices. A first step is to determine the Cox ring of the quotient space $\mathbb{C}^{4} / G$.

Proposition 3.1. The quotient $\mathbb{C}^{4} /[G, G] \rightarrow \mathbb{C}^{4} / G$ by the induced action of $G^{\prime}$ is the characteristic space over $\mathbb{C}^{4} / G$. The Cox ring $\mathcal{R}\left(\mathbb{C}^{4} / G\right) \cong \mathbb{C}\left[S_{1}, \ldots, S_{4}\right]^{[G, G]}$ is isomorphic to $\mathbb{C}\left[T_{1}, \ldots, T_{10}\right] / I$, where the ideal $I$ is generated by

$$
\begin{array}{ccc}
T_{5} T_{6}+T_{8} T_{9}-T_{7} T_{10}, & T_{4} T_{6}+T_{2} T_{7}+T_{1} T_{8}, & T_{3} T_{6}-T_{2} T_{9}-T_{1} T_{10}, \\
T_{2} T_{6}+T_{4} T_{7}+T_{3} T_{9}, & T_{1} T_{6}-T_{4} T_{8}-T_{3} T_{10}, & T_{5}^{2}-T_{7}^{2}+T_{9}^{2}+T_{2}^{2}, \\
T_{4} T_{5}+T_{1} T_{9}+T_{2} T_{10}, & T_{3} T_{5}-T_{1} T_{7}-T_{2} T_{8}, & T_{2} T_{5}+T_{3} T_{8}+T_{4} T_{10} \\
T_{1} T_{5}-T_{3} T_{7}-T_{4} T_{9}, & T_{4}^{2}-T_{6}^{2}-T_{9}^{2}+T_{10}^{2}, & T_{3} T_{4}+T_{7} T_{9}-T_{8} T_{10}, \\
T_{2} T_{4}+T_{6} T_{7}-T_{5} T_{10}, & T_{1} T_{4}-T_{6} T_{8}+T_{5} T_{9}, & T_{3}^{2}+T_{6}^{2}-T_{7}^{2}+T_{8}^{2}, \\
T_{2} T_{3}+T_{5} T_{8}-T_{6} T_{9}, & T_{1} T_{3}-T_{5} T_{7}+T_{6} T_{10}, & T_{2}^{2}-T_{6}^{2}-T_{8}^{2}+T_{10}^{2}, \\
T_{1} T_{2}+T_{7} T_{8}-T_{9} T_{10}, \quad T_{1}^{2}+T_{6}^{2}-T_{7}^{2}+T_{9}^{2}
\end{array}
$$

and the grading of $\mathbb{C}\left[T_{1}, \ldots, T_{10}\right] / I$ by $\mathrm{Cl}\left(\mathbb{C}^{4} / G\right) \cong \mathbb{X}\left(G^{\prime}\right) \cong(\mathbb{Z} / 2 \mathbb{Z})^{4}$ is given by assigning to the class of $T_{i}$ the ith column of the following matrix over $\mathbb{Z} / 2 \mathbb{Z}$ :

$$
Q:=\left[\begin{array}{llllllllll}
0 & 0 & 0 & 1 & 1 & 0 & 1 & 1 & 0 & 0 \\
0 & 0 & 0 & 1 & 0 & 1 & 0 & 0 & 1 & 1 \\
1 & 0 & 1 & 0 & 0 & 0 & 0 & 1 & 1 & 0 \\
0 & 1 & 1 & 0 & 0 & 0 & 1 & 0 & 0 & 1
\end{array}\right]
$$

The variables $T_{i}$ define pairwise non-associated $\mathbb{X}\left(G^{\prime}\right)$-prime elements in $\mathcal{R}\left(\mathbb{C}^{4} / G\right)$. The above presentation of the Cox ring gives rise to a commutative diagram

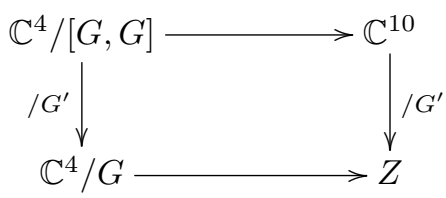

where the horizontal arrows are closed embeddings. The ambient affine toric variety $Z=$ $\mathbb{C}^{10} / G^{\prime}$ of $\mathbb{C}^{4} / G$ is of dimension ten and arises from the cone $\sigma \subseteq \mathbb{Q}^{10}$ generated by the vectors

$$
\begin{gathered}
v_{1}:=e_{1}, \quad v_{2}:=e_{2}, \quad v_{3}:=e_{3}, \quad v_{4}:=e_{4}, \quad v_{5}:=e_{5}, \\
v_{6}:=e_{1}+e_{2}+e_{5}+2 e_{6}, \quad v_{7}:=e_{7}, \quad v_{8}:=e_{4}+e_{5}+e_{7}+2 e_{8}, \\
v_{9}:=e_{1}+e_{3}+e_{4}+e_{5}+e_{7}+2 e_{8}, \quad v_{10}:=e_{1}+e_{2}+e_{7}+2 e_{10} .
\end{gathered}
$$

There are precisely five toric orbits of dimension six in $Z$ that contain singularities of $\mathbb{C}^{4} / G$. Any further singularities of $\mathbb{C}^{4} / G$ are contained in the closures of these orbits. The faces of $\sigma$ corresponding to these five toric orbits are:

$$
\begin{gathered}
\operatorname{cone}\left(v_{1}, v_{2}, v_{5}, v_{6}\right), \quad \operatorname{cone}\left(v_{4}, v_{5}, v_{7}, v_{8}\right), \quad \operatorname{cone}\left(v_{1}, v_{3}, v_{8}, v_{9}\right), \\
\operatorname{cone}\left(v_{4}, v_{6}, v_{9}, v_{10}\right), \quad \operatorname{cone}\left(v_{2}, v_{3}, v_{7}, v_{10}\right) .
\end{gathered}
$$


Proof. The first sentence is a direct consequence of a more general statement due to Arzhantsev and Gauffullin [3]. We verify the claimed presentation of the Cox ring

$$
\mathcal{R}\left(\mathbb{C}^{4} / G\right) \cong \mathbb{C}\left[S_{1}, \ldots, S_{4}\right]^{[G, G]} .
$$

The algebra of $[G, G]$-invariant polynomials is generated by the quadratic monomials of $\mathbb{C}\left[S_{1}, \ldots, S_{4}\right]$. Moreover, $G^{\prime}$ acts on the ten-dimensional $\mathbb{C}$-vector subspace generated by these monomials and the following $G^{\prime}$-homogeneous polynomials form a basis of this vector subspace:

$$
\begin{array}{ccc}
S_{1} S_{2}+S_{3} S_{4}, \quad S_{1} S_{2}-S_{3} S_{4}, & S_{1} S_{3}+S_{2} S_{4}, \\
S_{1} S_{3}-S_{2} S_{4}, \quad S_{1} S_{4}+S_{2} S_{3}, & S_{1} S_{4}-S_{2} S_{3}, \\
\frac{1}{2}\left(S_{1}^{2}+S_{2}^{2}+S_{3}^{2}+S_{4}^{2}\right), & \frac{1}{2}\left(-S_{1}^{2}-S_{2}^{2}+S_{3}^{2}+S_{4}^{2}\right), \\
\frac{1}{2}\left(-S_{1}^{2}+S_{2}^{2}-S_{3}^{2}+S_{4}^{2}\right), & \frac{1}{2}\left(S_{1}^{2}-S_{2}^{2}-S_{3}^{2}+S_{4}^{2}\right) .
\end{array}
$$

According to the order of listing, the weights of these polynomials occur as columns of the matrix $Q$ of the assertion. Thus, we have found ten $\mathbb{X}\left(G^{\prime}\right)$-homogeneous prime generators for $\mathbb{C}\left[S_{1}, \ldots, S_{4}\right]^{[G, G]}$

The desired presentation of the Cox ring is now obtained by sending $T_{i}$ to the $i$ th generator and the ideal $I$ is computed as the ideal of algebraic relations between the above generators. The remaining claims are direct applications of [2, Sections 3.2.5 and 3.3.1].

The second step is to compute the Cox $\operatorname{ring} \mathcal{R}(X)$ for a certain resolution $X \rightarrow \mathbb{C}^{4} / G$ of singularities. This is done using the technique of toric ambient modifications introduced in [15]: we just perform an evident partial toric resolution of the ambient affine toric variety $Z$ and show that the proper transform $X$ of $\mathbb{C}^{4} / G$ is as wanted. The 81 symplectic resolutions then correspond to the full-dimensional chambers of the Mori chamber decomposition of the cone of movable divisor classes of $X$.

Theorem 3.2. Consider the polynomial ring $\mathbb{C}\left[T_{1}, \ldots, T_{15}\right]$ with the $\mathbb{Z}^{5}$-grading assigning to the variables the columns of the following matrix:

$$
\left[\begin{array}{rrrrrrrrrrrrrrr}
1 & 1 & 0 & 0 & 1 & 1 & 0 & 0 & 0 & 0 & -2 & 0 & 0 & 0 & 0 \\
0 & 0 & 0 & 1 & 1 & 0 & 1 & 1 & 0 & 0 & 0 & -2 & 0 & 0 & 0 \\
1 & 0 & 0 & 0 & 0 & 1 & -1 & 0 & 1 & 0 & -1 & 1 & -1 & -1 & 1 \\
1 & 1 & 1 & -1 & 0 & 0 & 0 & 0 & 0 & 0 & -1 & 1 & -1 & 1 & -1 \\
0 & 1 & 0 & 0 & 0 & 1 & 0 & -1 & 0 & 1 & -1 & 1 & 1 & -1 & -1
\end{array}\right]
$$

and the ideal $I \subseteq \mathbb{C}\left[T_{1}, \ldots, T_{15}\right]$ generated by the following $\mathbb{Z}^{5}$-homogeneous polynomials:

$$
\begin{gathered}
T_{3} T_{6}-T_{2} T_{9}-T_{1} T_{10}, \quad T_{3} T_{5}-T_{1} T_{7}-T_{2} T_{8}, \quad T_{3} T_{4}+T_{7} T_{9}-T_{8} T_{10}, \\
T_{2} T_{4}+T_{6} T_{7}-T_{5} T_{10}, \quad T_{1} T_{4}-T_{6} T_{8}+T_{5} T_{9}, \\
T_{1} T_{8} T_{13}+T_{4} T_{6} T_{14}+T_{2} T_{7} T_{15}, \quad T_{5} T_{8} T_{12}-T_{6} T_{9} T_{14}+T_{2} T_{3} T_{15}, \\
T_{5} T_{7} T_{12}-T_{1} T_{3} T_{13}-T_{6} T_{10} T_{14}, \quad T_{5}^{2} T_{12}-T_{1}^{2} T_{13}-T_{6}^{2} T_{14}+T_{2}^{2} T_{15}, \\
T_{4} T_{5} T_{12}+T_{1} T_{9} T_{13}+T_{2} T_{10} T_{15}, \quad T_{6}^{2} T_{11}-T_{4}^{2} T_{12}+T_{9}^{2} T_{13}-T_{10}^{2} T_{15}, \\
T_{5} T_{6} T_{11}+T_{8} T_{9} T_{13}-T_{7} T_{10} T_{15}, \quad T_{2} T_{6} T_{11}+T_{4} T_{7} T_{12}+T_{3} T_{9} T_{13}, \\
T_{1} T_{6} T_{11}-T_{4} T_{8} T_{12}-T_{3} T_{10} T_{15}, \quad T_{5}^{2} T_{11}+T_{8}^{2} T_{13}+T_{4}^{2} T_{14}-T_{7}^{2} T_{15}, \\
T_{2} T_{5} T_{11}+T_{3} T_{8} T_{13}+T_{4} T_{10} T_{14}, \quad T_{1} T_{5} T_{11}-T_{4} T_{9} T_{14}-T_{3} T_{7} T_{15}, \\
T_{2}^{2} T_{11}-T_{7}^{2} T_{12}+T_{3}^{2} T_{13}+T_{10}^{2} T_{14}, \quad T_{1} T_{2} T_{11}+T_{7} T_{8} T_{12}-T_{9} T_{10} T_{14}, \\
T_{1}^{2} T_{11}-T_{8}^{2} T_{12}+T_{9}^{2} T_{14}-T_{3}^{2} T_{15} .
\end{gathered}
$$


Then the ideal $I$ is homogeneous with respect to the $\mathbb{Z}^{5}$-grading and, thus, the factor ring $R:=\mathbb{C}\left[T_{1}, \ldots, T_{15}\right] / I$ inherits a $\mathbb{Z}^{5}$-grading. Moreover, the following statements hold.

(i) The ring $R$ is factorial, the variables $T_{1}, \ldots, T_{15}$ define pairwise non-associated prime elements in $R$ and any fourteen of the degrees of the fifteen variables generate $\mathbb{Z}^{5}$ as a group.

(ii) The moving cone $\operatorname{Mov}(R)$ of the $\mathbb{Z}^{5}$-graded ring $R$ is of dimension five and contains precisely 81 five-dimensional geometric invariant theory (GIT) chambers. Each of these chambers defines a smooth variety $X_{i}$ having divisor class group $\mathrm{Cl}\left(X_{i}\right) \cong \mathbb{Z}^{5}$ and Cox ring $\mathcal{R}\left(X_{i}\right)=R$.

(iii) The subring $R_{0} \subseteq R$ of elements of degree zero is isomorphic to the coordinate ring of $\mathbb{C}^{4} / G$ and, thus, we have canonical morphisms $X_{i} \rightarrow \mathbb{C}^{4} / G$, each of which is a symplectic resolution of singularities.

Proof. We start with $X_{0}:=\mathbb{C}^{4} / G$ and the presentation of its Cox ring $R_{0}:=\mathcal{R}\left(X_{0}\right)$ given in Proposition 3.1. We enter the relations of $R_{0}$ given there as a list RLO in MDSpackage and as well the grading matrix, which we now call Q0mat. Using these data, we then define the degree map $Q_{0}: \mathbb{Z}^{10} \rightarrow(\mathbb{Z} / 2 \mathbb{Z})^{4}$, the Cox ring $R_{0}$ and $X_{0}$ :

$$
\begin{gathered}
>\mathrm{Q} 0:=\operatorname{createAGH}(\operatorname{createAG}(10), \operatorname{createAG}(0,[2,2,2,2]), \mathrm{Q} 0 \mathrm{mat}) ; \\
Q 0:=A G H([10,[]],[0,[2,2,2,2]]) \\
>\mathrm{R} 0:=\operatorname{createGR}(\mathrm{RLO}, \operatorname{vars}(10),[\mathrm{Q} 0], \text { 'Singular' }) ; \\
R 0:=G R(10,20,[0,[2,2,2,2]]) \\
>\mathrm{X} 0:=\operatorname{createMDS}(\mathrm{RO},[0]) ; \\
X 0:=\operatorname{MDS}(10,20,4,[0,[2,2,2,2]]) .
\end{gathered}
$$

We resolve the singularities of $X_{0}$ via a suitable toric modification of the ambient affine toric variety $Z$. By Proposition 3.1, there are five singular toric orbits, the closures of which form the singular locus of $Z$. The first evident step towards any toric resolution is to resolve the corresponding cones. This is achieved in each of the five cases by a single barycentric subdivision, that is, we insert five new rays $\mathbb{Q}_{\geqslant 0} \cdot v_{1}, \ldots, \mathbb{Q} \geqslant 0 \cdot v_{5}$ in total, where the list of $v_{i}$ is

$$
\begin{gathered}
>\mathrm{VL}:=[[1,1,0,0,1,1,0,0,0,0],[0,0,0,1,1,0,1,1,0,0], \\
[1,0,1,1,1,0,1,1,1,0],[1,1,1,1,1,1,1,0,1,1],[0,1,1,0,0,0,1,0,0,1]] ; \\
V L:= \\
{[[1,1,0,0,1,1,0,0,0,0],[0,0,0,1,1,0,1,1,0,0],[1,0,1,1,1,0,1,1,1,0],} \\
[1,1,1,1,1,1,1,0,1,1],[0,1,1,0,0,0,1,0,0,1]] .
\end{gathered}
$$

The Cox ring $R$ of the proper transform $X$ of $X_{0}$ under such a partial ambient resolution can be computed via the command MDSmodify, which implements [18, Algorithm 3.6]:

$>\mathrm{X}:=$ MDSmodify(XO, VL, 'Singular', 'verify');

Verification successful: all variables are prime.

$$
X:=\operatorname{MDS}(15,20,4,[5,[]])
$$

$>\mathrm{R}:=\operatorname{MDSdata}(\mathrm{X})[1]$

$$
R:=G R(15,20,[5,[]])
$$


The algorithm thus returns the $\mathbb{Z}^{5}$-graded ring $\mathrm{R}$ as the Cox ring $R:=R_{2}$ of the assertion and, additionally, verifies the statements of the first assertion. For the second assertion, we compute the GIT fan using MDSpackage's implementation of [25, Algorithm 8]:

$>F:=\operatorname{GRgitfan}(R$, 'fan');

$$
F:=F A N(5,0,[0,0,0,0,207])
$$

$>\operatorname{Mov}:=\operatorname{MDSmov}(\mathrm{X})$;

$$
\text { Mov }:=\operatorname{CONE}(5,5,0,5,5) .
$$

The command $U:=\operatorname{select}(c->\operatorname{contains}(\operatorname{Mov}, \mathrm{C})$, maximal $(\mathrm{F})$ ) retrieves the 81 fulldimensional chambers inside the moving cone, as claimed. We test smoothness of the varieties $X_{i}$ associated to the chambers U[i] using an MDSpackage function based on the criterion [2, Corollary 3.3.1.12]; compare [12] for a related approach:

$>\mathrm{w} 1:=\operatorname{relint}(\mathrm{U}[1])$

$$
w 1:=[6,1,3,3,3]
$$

$>\mathrm{X} 1:=\operatorname{createMDS}(\mathrm{R}, \mathrm{w} 1)$;

$$
X 1:=\operatorname{MDS}(15,20,4,[5,[]])
$$

> MDSissmooth(X1, 'magma');

\section{true}

All test results are positive. The computation is sped up a bit by calling magma under the surface; it takes some hours on a personal computer. Note that $X$ is among the $X_{i}$. The third assertion is clear by the way we obtained the common Cox ring $R$ of the $X_{i}$.

REMARK 3.3. Having proved Theorem 3.2, a discussion of the geometry of the resolutions is possible via the methods of MDSpackage.

Acknowledgement. We are grateful to Jarosław Wiśniewski for informing us about the current state of research concerning the 81 symplectic resolutions just discussed and encouraging us to work out Theorem 3.2.

\section{References}

1. M. Artebani and A. Laface, 'Hypersurfaces in Mori dream spaces', J. Algebra 371 (2012) 26-37.

2. I. Arzhantsev, U. Derenthal, J. Hausen and A. Laface, Cox rings, Cambridge Studies in Advanced Mathematics 144 (Cambridge University Press, Cambridge, 2015).

3. I. Arzhantsev and S. Gă̌fullin, 'Cox rings, semigroups, and automorphisms of affine varieties', Math. Sb. 201 (2010) no. 1, 3-24.

4. B. Bechtold, J. Hausen, E. Huggenberger and M. Nicolussi, 'On terminal Fano 3-folds with 2-torus action', Int. Math. Res. Not. IMRN, doi:10.1093/imrn/rnv190.

5. G. Bellamy and T. Schedler, 'A new linear quotient of $\mathbf{C}^{4}$ admitting a symplectic resolution', Math. Z. 273 (2013) no. 3-4, 753-769.

6. F. Berchtold and J. Hausen, 'Cox rings and combinatorics', Trans. Amer. Math. Soc. 359 (2007) no. 3, 1205-1252.

7. F. Berchtold, J. Hausen, S. Keicher, R. Vollmert and M. Widmann, 'TorDiv 1.4 - a Maple package on toric geometry and geometric invariant theory', available at http://www.mathematik. uni-tuebingen.de/ hausen/. 
8. C. Birkar, P. Cascini, C. Hacon and J. McKernan, 'Existence of minimal models for varieties of log general type', J. Amer. Math. Soc. 23 (2010) 405-468.

9. W. Bosma, J. Cannon and C. Playoust, 'The Magma algebra system. I. The user language', J. Symbolic Comput. 24 (1997) no. 3-4, 235-265.

10. G. Brown, 'A database of polarized K3 surfaces', Experiment. Math. 16 (2007) no. 1, 7-20.

11. U. Derenthal, 'Singular Del Pezzo surfaces whose universal torsors are hypersurfaces', Proc. Lond. Math. Soc. (3) 108 (2014) no. 3, 638-681.

12. M. Donten-Bury and J. Wiśniewski, 'On 81 symplectic resolutions of a 4-dimensional quotient by a group of order 32', Preprint, 2014, arXiv:1409.4204v3.

13. M. Franz, 'Convex - a Maple package for convex geometry', available at http://www.math.uwo.ca/ $\sim$ mfranz/convex/ and the Maple homepage http://www.maplesoft.com/applications/view.aspx?SID= 4507.

14. S. Gutsche, 'GAP package ToricVarieties', available at http://www.gap-system.org/Packages/ toricvarieties.html.

15. J. Hausen, 'Cox rings and combinatorics II', Mosc. Math. J. 8 (2008) no. 4, 711-757, 847.

16. J. HAusen and S. Keicher, 'Cox ring database', available at http://www.math.uni-tuebingen.de/ user/keicher/coxringdb/.

17. J. HAUSEN and S. Keicher, 'MDSpackage - a package for Mori dream spaces', available at http://www.math.uni-tuebingen.de/user/keicher/MDS/.

18. J. Hausen, S. Keicher and A. Laface, 'Computing Cox rings', Math. Comp. 85 (2016) no. 297, $467-502$.

19. Y. Hu and S. KeEL, 'Mori dream spaces and GIT', Michigan Math. J. 48 (2000) 331-348; dedicated to William Fulton on the occasion of his 60th birthday.

20. A. R. IANo-Fletcher, 'Working with weighted complete intersections', Explicit birational geometry of 3-folds, London Mathematical Society Lecture Note Series 281 (Cambridge University Press, 2000) $101-173$.

21. S.-Y. Jow, 'A Lefschetz hyperplane theorem for Mori dream spaces', Math. Z. 268 (2011) no. 1-2, 197-209.

22. D. JoYner, 'GAP package toric', available at http://www.gap-system.org/Packages/toric.html.

23. L. Kastner, B. Lorenz, A. Paffenholz and A.-L. Winz, polymake_toric, available at https://github.com/lkastner/polymake_toric.

24. S. KeIcher, 'Computing the GIT-fan', Int. J. Algebra Comput. 22 (2012) no. 7, 1250064, 11 pp.

25. S. KeICher, 'Algorithms for Mori dream spaces', PhD Thesis, Universität Tübingen, 2014, http://nbn-resolving.de/urn:nbn:de:bsz:21-dspace-540614.

26. MAPle 10, Maplesoft, a division of Waterloo Maple Inc., Waterloo, Ontario. See http://www.maplesoft.com/.

27. J. McKernan, 'Mori dream spaces', Jpn. J. Math. 5 (2010) no. 1, 127-151.

28. H. Verrill and D. Joyner, 'Computing with toric varieties', J. Symbolic Comput. 42 (2007) no. 5, $511-532$.

Jürgen Hausen

Mathematisches Institut

Universität Tübingen

Auf der Morgenstelle 10

72076 Tübingen

Germany

juergen.hausen@uni-tuebingen.de

\author{
Simon Keicher \\ Mathematisches Institut \\ Universität Tübingen \\ Auf der Morgenstelle 10 \\ 72076 Tübingen \\ Germany
}

keicher@mail.mathematik.uni-tuebingen.de 